\title{
A novel method of rejecting muon backgrounds for the detection of the highest energy neutrinos
}

\author{
The IceCube Collaboration* \\ http://icecube.wisc.edu/collaboration/authors/icrc19_icecube \\ E-mail: lu.lu@icecube.wisc.edu
}

\begin{abstract}
The IceCube neutrino observatory, located at the South Pole, has discovered the astrophysical diffuse flux and multi-PeV neutrino events. The $10-100 \mathrm{PeV}$ energy range is key to test the possible common origin of IceCube neutrinos and ultra-high energy cosmic rays. In this work, we present a new analysis targeting at cascade-like showers of deposit energy 10-100 PeV. The new event selection is sensitive to both contained and partially-contained cascades.
\end{abstract}

Corresponding authors: $\mathrm{Lu} \mathrm{Lu}^{\dagger 1}$, Christian Haack ${ }^{2}$, Tianlu Yuan ${ }^{3}$

${ }^{1}$ Department of Physics, Faculty of Science, Chiba University, Japan

2 III. Physikalisches Institut B, RWTH Aachen University, Germany

${ }^{3}$ Dept. of Physics and Wisconsin IceCube Particle Astrophysics Center, University of Wisconsin, Madison, USA

36th International Cosmic Ray Conference -ICRC2019-

July 24th - August 1st, 2019

Madison, WI, U.S.A.

\footnotetext{
${ }^{*}$ For collaboration list, see PoS(ICRC2019) 1177.

${ }^{\dagger}$ Speaker.
} 
The origin of ultra-high energy cosmic rays (UHECR) is among one of the most enduring mysteries in science and still unsolved after more than 100 years of their discovery. The uncertainties in hadronic interaction models and extragalactic magnetic fields make it difficult to reveal their sources with cosmic-ray data. However, neutrinos are chargeless and weakly interacting, which make them ideal messengers to study the extreme environments in the source region.

Neutrinos could be produced at the acceleration site from cosmic-ray interaction with the ambient gas or radiation. Their flavour can be used to differentiate production mechanisms, e.g. to study the large magnetic fields which are required by muon-damped sources. In addition, since there is no time delay for neutrino propagation one could study cosmic-ray sources in real-time which is not possible with diffused cosmic-rays. The IceCube Neutrino Observatory, located at the geographic South Pole, was completed in late 2010 and has been measuring high-quality data with greater than $99 \%$ up-time. The astrophysical spectrum has been measured $[1,2]$ and the first possible cosmic-ray source has been reported thanks to the real-time multi-messenger observations $[3,4]$. However, there is no evidence for correlations of neutrinos measured by IceCube with blazars observed by Fermi gamma-ray telescope [5, 6], high-luminosity GRBs [7], star burst galaxies [8] or the Galactic plane [9]. The dominant origin for the observed high-energy neutrinos component is yet unknown.

Intriguingly, measurements of the diffuse astrophysical neutrino flux shows that the neutrino energy density at $\sim \mathrm{PeV}$ is at the same order of magnitude as the energy density of UHECR at $\sim 10 \mathrm{EeV}$. Unification models, which explain both IceCube neutrinos and UHECR, have been proposed to explain this coincidence $[10,11,12,13]$. Precise measurements of the diffuse neutrino flux at $\sim 10 \mathrm{PeV}$ to identify a possible break, cut-off or a new component are needed to test the unification theories. In this proceeding, we will first summarise the existing IceCube event selections designed to search for high-energy hadronic or electromagnetic shower events, then we discuss how to effectively reject the cosmic-ray muon background by using features of hadronic showers.

\section{Current shower detection with IceCube}

Electromagnetic or hadronic shower events, also known as cascade events can be generated via neutrino-nucleon interactions such as neutral-current neutrino interactions, (anti-)electron neutrino charged-current interactions and a fraction of (anti-)tau charged-current interactions. In addition, the Glashow resonance [14], which is the resonant scattering of anti-electron neutrino interacting with atomic electrons can also result in cascade features. Compared with track-like events produced by high-energy muons, showers have better neutrino energy resolution of about $10-20 \%$ instead of $\sim 50 \%$ at PeV neutrino energy which helps to determine fine structures of the diffuse flux. So far the highest energy cascade event was found in the PeV Energy Partially-contained Event (PEPE) channel [15]. Its event view is shown in Fig. 1a. The event has a deposited energy of $\sim 6 \mathrm{PeV}$ and is the first Glashow-resonance candidate. More details will be included in a publication elsewhere.

The PEPE analysis was designed to select events that failed the veto criteria of the High Energy Start Events (HESE) [1] selection. Furthermore the event selection requires greater than $80 \%$ of the total charge deposited in the outer two rows of strings. The boundary to mark the outer region is shown in Fig. 1b. A Boosted Decision Tree (BDT) [17] was used to classify multi-PeV (anti- 


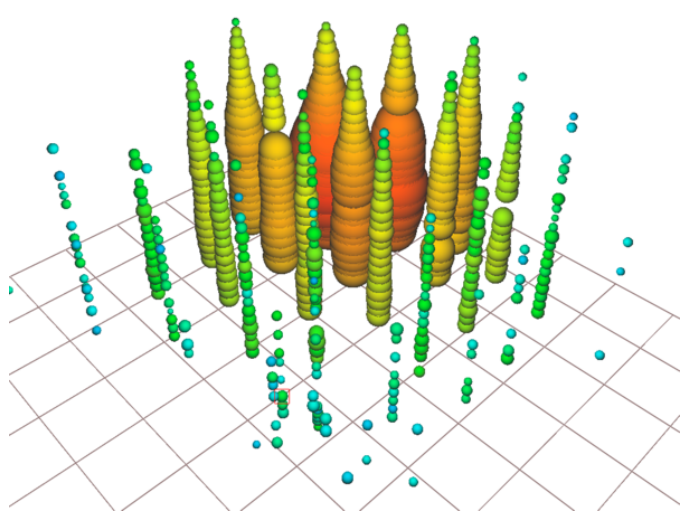

(a) $1 \mathrm{a}$

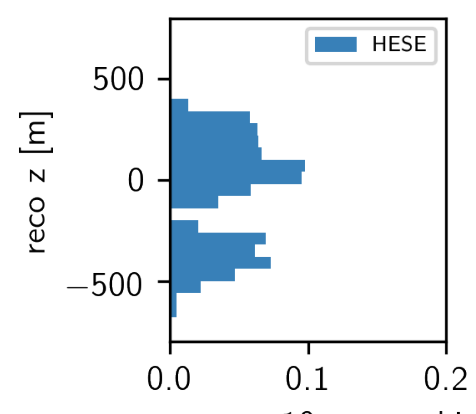

event per 10 yrs per bin

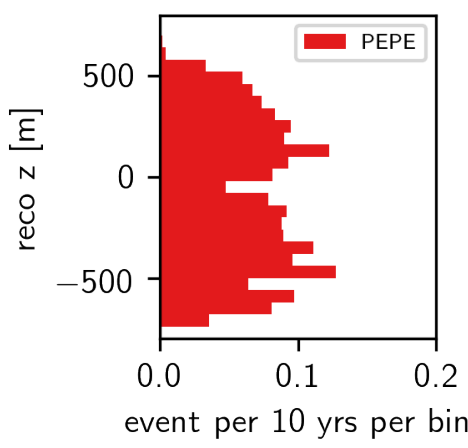

(c) $1 \mathrm{c}$

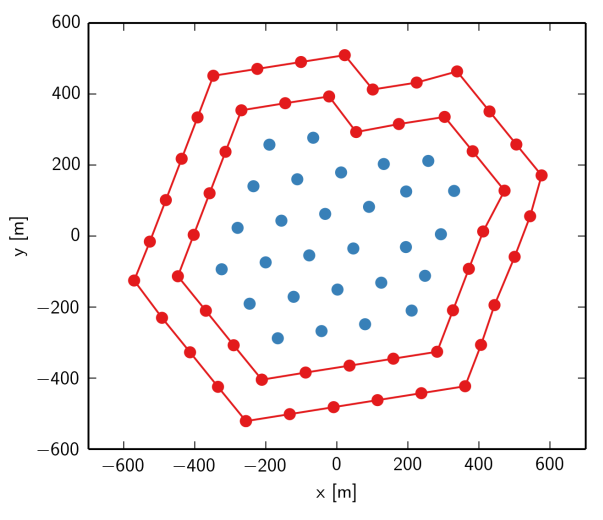

(b) $1 \mathrm{~b}$

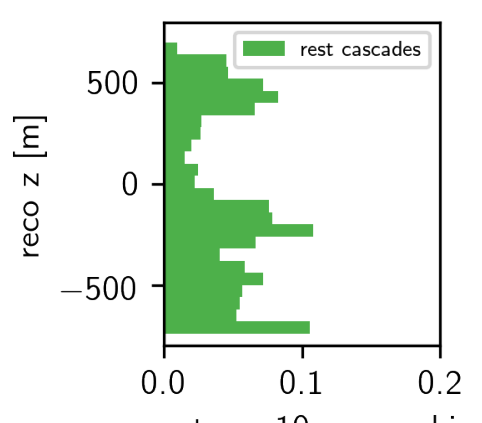

event per 10 yrs per bin

Figure 1: (a) Event view of the Glashow resonance candidate found in the PEPE analysis. (b) Definition of outer strings used in the PEPE analysis. The red line outlines the boundary of inner and outer. (c) Distribution of reconstructed depth $\mathrm{z}$ for cascade events selected by HESE (blue), PEPE (red) and those not selected (green). The histograms are normalised to 10-year expectation of $v_{e}$ and $\bar{v}_{e}$ with $E_{v} \geq 8 \mathrm{PeV}$, weighted by best-fit from [16]

)electron neutrinos from cosmic-ray background. As a result, there are two types of high-energy shower events not selected by HESE or PEPE:

- Interaction vertex above/below the IceCube detectors or located at the dust layer

- Interaction vertex outside the detector and more than $150 \mathrm{~m}$ away from the closest edge string leading to a BDT score below the threshold of 0.5 in the PEPE selection.

Distributions of the vertex depth $z$ for $v_{e} / \bar{v}_{e}$ with energies greater than $8 \mathrm{PeV}$ are shown in Fig. 1c. The events are reconstructed using a maximum likelihood-based method including timing information assuming cascade hypotheses. Events selected by HESE are concentrated in the centre of the detector with the exception of the dust layer. PEPE selects a factor of $\sim 2$ more compared with HESE. The rest of the events that have been successfully reconstructed (reduced likelihood for cascades less than 10) are most abundant on the top, bottom, and dust layer of the detector. 
To expand the PEPE analysis to include partially-contained cascades with interaction vertices within or above the top veto-layer of the detector, the biggest issue is the cosmic-ray muon background. In the next session we describe a new method to reduce such backgrounds.

\section{A new search with better cosmic-ray background rejection}

The background of $v_{e} / \bar{v}_{e}$ searches which have interaction vertex outside of the detector are muons produced in cosmic-ray showers. To mimic single cascades the cosmic-ray event needs to have stochastic energy losses close to the edge of the detector without light yields which are inconsistent with a cascade hypothesis. The BDT trained for the original PEPE includes features to describe timing information of each event, such as the time difference between the first hit sensor with the sensor of highest charges. Although such parameters are efficient, they do not contain full timing information of all sensors. Here we demonstrate two possible improvements for the cosmic-ray muon background rejection starting from the differences of muons seen in cosmic-ray showers and neutrino interactions.

\subsection{Muons from cosmic-ray background}

High-energy cosmic-ray muons penetrate through the earth usually reach the detector composed of a bundle of muons. $\mathrm{A} \sim 10 \mathrm{PeV}$ single muon at the detector correspond to $100 \mathrm{PeV}-1 \mathrm{EeV}$ muon energy on the ground depending on the zenith angle of the shower and the depth of the muon where it reaches the detector. On average a muon of $10 \mathrm{PeV}$ can travel more than $10 \mathrm{~km}$ in ice and $\sim 100 \mathrm{~m}$ for a $\sim 20 \mathrm{GeV}$ muon. It means that it is extremely unlikely for a $10 \mathrm{PeV}$ muon to lose all its energy within $\sim 20 \mathrm{~m}$ but instead it prefers to propagate to a distance much larger than the diameter of IceCube. This is the first key difference with how energies are deposited for neutrino interactions and will be discussed in section 2.2.

Another unique feature of cosmic-ray mimicked cascades is the photon hits which happen before the supposed neutrino interaction. An illustration is shown in Fig.2. The brightest sensor of the event is closest to a stochastic energy loss along the muon track, while prior to that muon energy losses are caught on a neighbouring string.

\subsection{Muons from neutrino interactions}

Hadronic showers are formed from neutrino-nucleon deep inelastic scatterings for which the energies of the hadronic jets depends on the inelasticity of the interaction. Muons are predominately produced from decays of mesons from the shower development and the energy distribution of muons is found to follow a power-law [18], namely

$$
\frac{d N_{\mu}}{d E}=A\left(\frac{E_{0}}{\mathrm{GeV}}\right)^{\kappa} \cdot\left(\frac{E_{\mu}}{\mathrm{GeV}}\right)^{-2-\kappa},
$$

where $E_{0}$ is the energy of the primary particle, $E_{\mu}$ is the muon energy, $\kappa \approx 1$, and $A \approx 3.5 \times 10^{-3} \mathrm{GeV}^{-1}$. Therefore, a hadronic shower with initial energy $1 \mathrm{PeV}$ is expected to be accompanied by multiple muons with energies larger than $10 \mathrm{GeV}$. A cumulative probability distribution for the highest energy muon from $>8 \mathrm{PeV} v_{e}$ and $\bar{v}_{e}$ is shown in Fig. 3. 


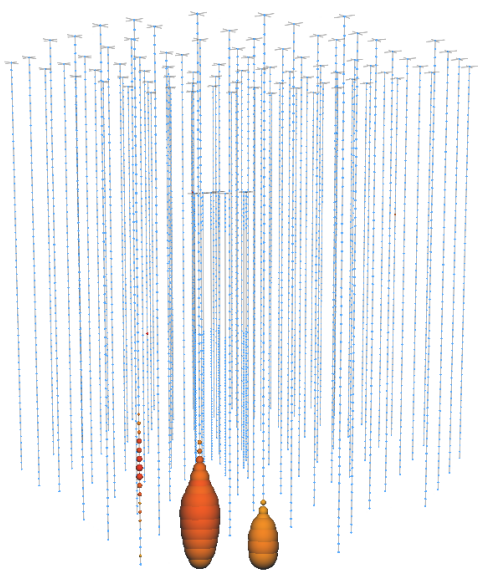

(a) $3 \mathrm{a}$

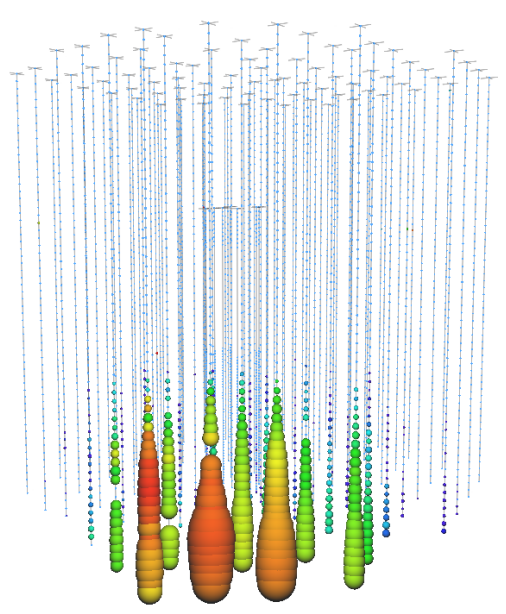

(b) $3 b$

Figure 2: An example of a cosmic-ray background event. The earliest hits are shown in red and the latest are in blue with green in between. Panel (a) and (b) show the same event but viewed at different time slice. Although the event appears to be similar with a neutrino-induced single cascade based on the topology integrated over all times, the first hit sensors yield an inconsistent starting times with cascade hypothesis.

The $\sim 80 \%$ highest energy cascades are expected to have a typical leading-muon energy $\leq 150 \mathrm{GeV}$, which corresponds to a mean-free-path of less than $1000 \mathrm{~m}$. It is possible to reconstruct an upper limit of the leading-muon energy by identifying the early sensor hits that are too early to be compatible with a cascade hypothesis. An example is illustrated in Fig. 4 showing a $24 \mathrm{PeV}$ electron neutrino undergoing a charged-current interaction and resulting in a leading-muon of energy $\sim 200 \mathrm{GeV}$. The interaction vertex is $\sim 50 \mathrm{~m}$ above the highest sensor close to string 46 . An $x-y$ projection of the event is shown in Fig. 5.

Muons in the hadronic cascade travel in straight lines with almost speed of light in vacuum while Cherenkov photons are scattered propagating in ice with speed of light in the medium. Consequently, sensors that are nearby the trajectories of the muons can be lit up by direct-Cherenkov photons from muons ahead of delayed photons generated from the electromagnetic (EM) shower close to the vertex position. Each small panel in Fig. 5 includes a waveform collected by a sensor. The leading muon passes by string 27 and 11 deposits charge from muon energy losses (in red) much earlier than the earliest possible allowed start time under the assumption of a spherical wavefront (green dashed line), which is determined by the vertex position of the interaction and independent of the cascade direction.

Once identified the early pulses, they can be separated from the rest of the EM-induced signals for a dual reconstruction.

\subsection{Dual reconstruction including muon information}

The early muon signals are small compared with the EM signals but they carry direct Cherenkovphoton information which is useful to improve the cascade direction reconstruction. A track hy- 


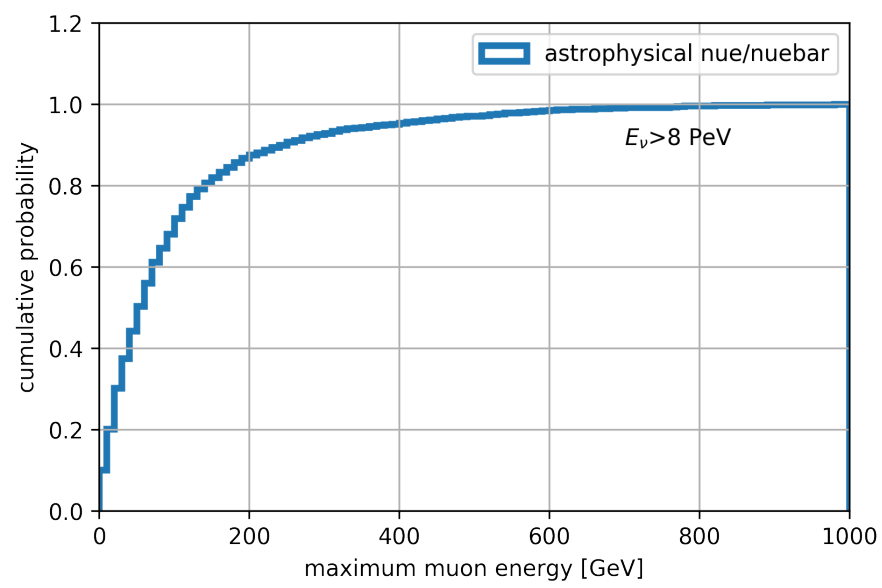

Figure 3: Cumulative probability distribution for maximum muon energy from astrophysical neutrinos over $8 \mathrm{PeV}$. The parameterisation of muons from hadronic showers is based on proton interacting with salt water and simulated via QGSJetII01 model in a modified CORSIKA software. Updates using more updated hadronic interaction models are work-in-progress.

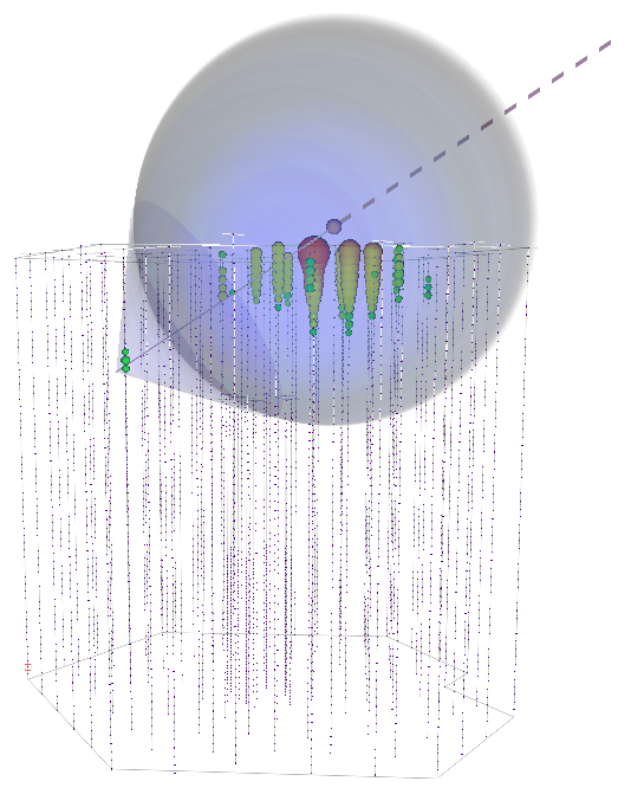

Figure 4: An example of a partiallycontained event which is not selected by the PEPE analysis. The primary electron neutrino has $24 \mathrm{PeV}$ with a leading muon from hadronic shower of $\sim 200 \mathrm{GeV}$. The Cherenkov cone led by the muonic component is ahead of the spherical photon light front. The dashed line shows the trajectory of the primary neutrino propagation before interacting just above the detector.

pothesis assuming a muon at position $\vec{x}$ at time $t$ is fitted for the early pulses:

$$
\vec{x}\left(t \mid \vec{d}, t_{0}, \vec{x}_{0}\right)=\vec{x}_{0}+\left(t-t_{0}\right) \cdot \vec{d},
$$

where $\vec{x}_{0}$ is the position at time $t_{0}$ and $\vec{d}$ the direction of the track. The likelihood model is based on the time arrival distributions of photons emitted from the track at each photon sensor:

$$
\mathscr{L}=\prod_{i}^{N_{\text {Sensor }}} \prod_{j}^{N_{\text {Photons }}^{i}} p_{i}\left(t_{j} \mid H\right),
$$



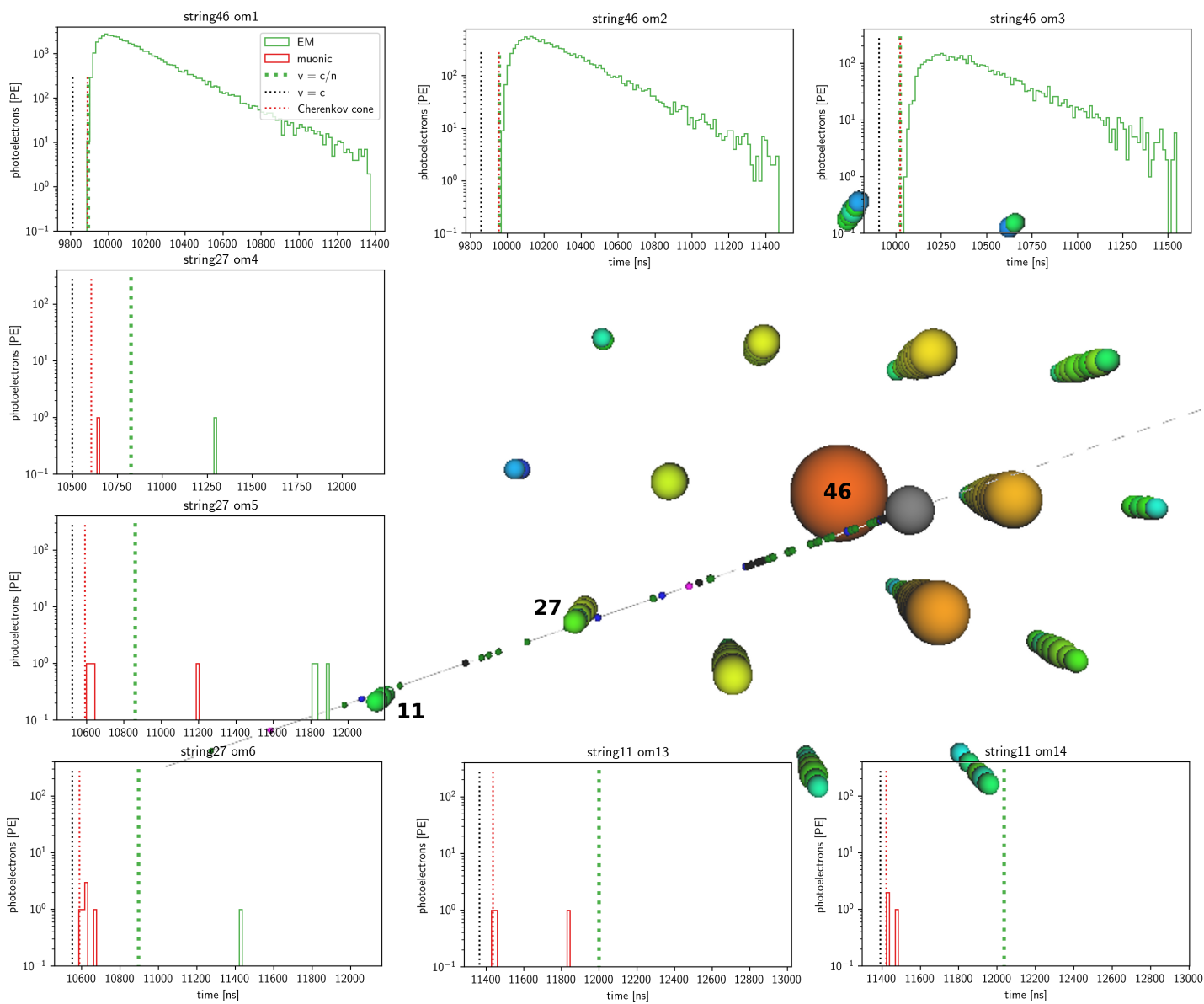

Figure 5: The $x-y$ projection of the same event in Fig.4. String 46 is closest to the neutrino interaction vertex position and the $\sim 200 \mathrm{GeV}$ muon travels passing by string 27 and 11 . Each panel shows arrival time distribution for charges due to electromagnetic (green) and muonic (red) component from the showers. The dashed lines indicate the earliest possible signal if particles travel in straight lines with speed of c (black), c/n (green) or following the Cherenkov path (red).

where $p_{i}$ is the photon arrival time distribution for sensor $i$ for an assumed track hypothesis $H$. Te arrival time distribution is obtained from lookup-tables which takes ice properties into account.

A comparison of a directional reconstruction using early muon signals with a reconstruction using the EM information is shown in Fig. 6. The uncertainties of the reconstruction are reduced significantly when using the early muon information. Note however that uncertainties regarding transverse momentum spread of the low-energy muons produced in the hadronic cascade compared to the incident neutrino direction are not included. The algorithm for the dual-reconstruction is still under development, potentially it can be implemented as a real-time trigger for multi-messenger followups for $\sim 10 \mathrm{PeV}$ transient searches.

\section{Conclusion and outlook}

A novel method to reject high-energy muons from cosmic-ray background has been proposed. 


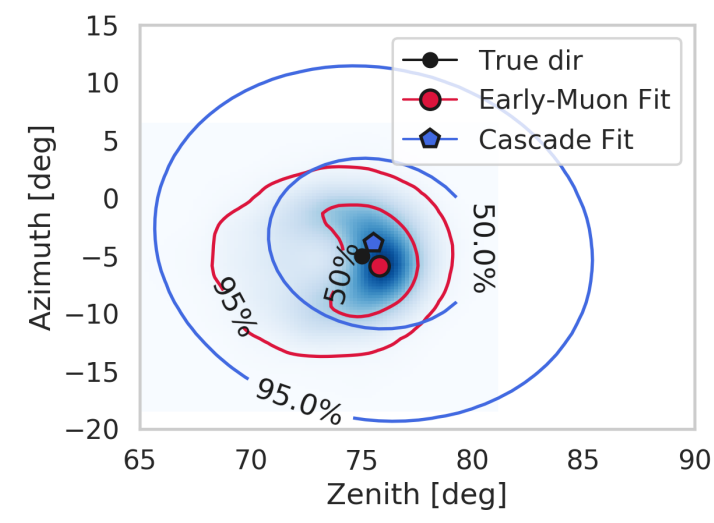

Figure 6: Angular reconstruction performance of the early muon reconstruction in comparison to the shower (cascade) only reconstruction for a multi-PeV hadronic shower. The blue-shaded region indicates the posterior probability density. The lines indicate $50 \%$ and $90 \%$ highest-probability density credible regions.

It can be applied to look for non-physical signals that are too early to explain a single hadroniccascade topology, which is expected from a neutrino-induced interaction. The new method also applies a dual (cascade and track) reconstruction to calculate the leading muon energy from a hadronic cascade. It reject events with leading muon energies greater than $200 \mathrm{GeV}$, which is efficient to remove cosmic-ray background but keeps $\geq 80 \%$ of $v_{e}$ and $\bar{v}_{e}$ of neutrino energy $>8 \mathrm{PeV}$. In the future, the new algorithm will be combined with the BDT scores from the PEPE analysis. We will also apply the new selection to data for a dedicated search for $\sim 10 \mathrm{PeV}$ neutrinos.

\section{References}

[1] IceCube Collaboration, Science 342 (2013) 1242856.

[2] IceCube Collaboration, The Astrophysical Journal 833 (2016) 3.

[3] IceCube Collaboration, Science 361 (2018) eaat1378.

[4] IceCube Collaboration, Astroparticle Physics 92 (2017) 30-41.

[5] IceCube Collaboration, The Astrophysical Journal 835 (2017) 45.

[6] IceCube Collaboration, Physical Review D 98 (2018) 062003.

[7] R. Abbasi, Y. Abdou, T. Abu-Zayyad, et al., Nature 484 (2012) 351.

[8] K. Bechtol, M. Ahlers, M. Di Mauro, et al., The Astrophysical Journal 836 (2017) 47.

[9] IceCube Collaboration, The Astrophysical Journal 849 (2017) 67.

[10] K. Fang and K. Murase, Nature Physics 14 (2018) 396.

[11] B. T. Zhang, K. Murase, S. S. Kimura, et al., Physical Review D 97 (2018) 083010.

[12] R.-Y. Liu, X.-Y. Wang, S. Inoue, R. Crocker, and F. Aharonian, Physical Review D 89 (2014) 083004.

[13] D. Boncioli, D. Biehl, and W. Winter, The Astrophysical Journal 872 (2019) 110.

[14] S. L. Glashow, Physical Review 118 (1960) 316.

[15] L. Lu, PoS (2017) 1002.

[16] L. Mohrmann, PoS (2015) 1066.

[17] Y. Freund and R. E. Schapire, Journal of computer and system sciences 55 (1997) 119-139.

[18] L. Rädel, Master's thesis, RWTH Aachen (2012). 